\title{
On the Learning of geometric concepts using Dynamic Geometry Software
}

\author{
Alex Sandro Gomes1 and Gerard Vergnaud2 \\ 1Centro de Informática Universidade Federal de Pernambuco (UFPE) \\ Caixa Postal 7851 - 50.740-540 - Recife - PE - Brasil \\ asg@cin.ufpe.br \\ 2 Laboratoire "Cognition et Activitès Finalisèes", Universitè Paris VIII - Saint-Denis \\ vergnaud@univ-paris8.fr
}

\begin{abstract}
Many results on computer mediated geometry learning conclude about different heuristic approaches to problem solving with Dynamic Geometry Software (DGS). However, little is described concerning conceptualization process. We used a theoretical framework built upon constructivist foundations for analyzing mediated learning of specific geometrical concepts. Our point is illustrated in a case study in which we analyzed studentsí interaction with a DGS. Our results points to a clear mapping of potential conceptualization of geometry in software using.
\end{abstract}

Key Words: Dynamic Geometry Software (DGS), Dynamic geometry, Conceptualization; Instrumental Genesis.

\section{Introduction}

At the end of the 80's the first systems of dynamic geometry appear: software conceived for the specific teaching and learning of plane geometry, comprising tools to allow the users to manipulate shapes on the computer screen directly and dynamically. According to the bibliography the results show that these Dynamic Geometry Software (DGSs) are rather efficient in the learning of geometry. Clements and Battista (1992) write "several findings are intriguingly consistent across studies using different computer environments. First, researchers and teachers consistently report that in such context students cannot hide what they do not understand (...). Second, at least at the high school level, students can become confused regarding the purpose of different components of a course; a single location for computer work, discussion, and lecture may alleviate this confusion (...). Third, evaluation of learning in such environments must be reconsidered, as traditional approaches did not assess the full spectrum of what was learned; in some cases, these approaches made little sense." (p.454). The aim of this paper is to present both theoretical framework and method allowing to describe emerging conceptualization in the use of Dynamic Geometry Software. 
Yerushalmy and Chazan (1992) analyse the use of the Geometric Supposer software. This system allows the students to draw, starting from the enunciation of the geometric properties; the students are no longer dependent on implicit inferences associated to the manipulations during the construction. The main point here is that the learning of geometrical concepts would be made easier by the fact that the constructions take place in a direct way, starting from the enunciation of the geometric properties of the drawing. The users actions are based upon formal geometrical aspects.

Geometry Supposer allows the choice of a primitive form, such as a triangle or a square (depending on the specific program), and of operations to modify the size and form of the figure. The software registers the sequence of constructions, and can apply it to other triangles and squares automatically. According to several experiments, Geometry Supposer can be effectively useful for the learning of Geometry (Clements and Battista, 1992, p. 452).

Cabri Géomètre appeared in a different institutional context (Laborde \& Vergnaud, 1994). The initial research work takes it as a tool in the teaching-learning process and considers it as an integral part of the didactical environment. According to their authors, this software tool presents strong idiosyncrasies, which interact with the studentsí activity and increase their potential learning. The computer performs a specific mediation of knowledge. Cabri evidences a distinction usually forgotten in the teaching of geometry: the distinction between the drawing and the figure. As Duval puts it, "the figure is an object belonging to theory whereas the drawing is a modelling on a sheet of paper, a computer screen or on the sand; the drawing is a model of the figure" (Duval, 1990). Laborde (1992) considers that, in Cabri "Drawings are no longer stereotypes, critical cases can be visualised, a geometrical situation can be considered, a necessary condition drawing preserving the expected properties.” (p.129).

The figure corresponds to a class of objects that keep certain properties invariant: "Another element of the complexity of the notion of figure derives from its intended and implicit generality: a figure does not refer to one object but to an infinity of objects. What is invariant in this class of objects are the relations between the objects." (p. 128). Laborde and Capponi (1994) adds that the geometrical figure consists in a reference attributed to all the drawings, it is then defined as a set of pairs formed by two terms: the first term being the reference, and the second one the set of drawings that exemplify it.

The specific contribution of Cabri is that it allows inserting into the learning process the variant aspects of a figure through the visualization of several drawings, which have the same 2 
geometrical properties. However, in the construction, the drawing is not of the same type of the one drawn on a piece of paper; consequently, the concepts formed by the learner, due to certain functionalities specific of Cabri are also distinct from the geometric objects envisaged: the drawings constructed with Cabri do not have the same characteristics as those coming from the use of the ruler and compasses: it is not the same kind of figure, as Cabri offers the possibility of transforming and varying the drawings while conserving the invariant characteristics. According to Laborde \& Capponi (1994), the conservation of the relevant properties through displacement is the main feature of Cabri, the one that facilitates the development of geometrical concepts. Some properties are added to geometrical objects. A displaced segment is not a segment anymore since a segment does not possess the property of being handled. Observing 6 th and 7 th grade students solving problems using Cabri, the authors Laborde \& Capponi (1994) noticed the absence of any implication between an empirical attempt (relying upon no geometric analysis), and the studentís progressive awareness of the need to have recourse to geometry to solve the drawing problem.

Hölz (1996) showed that the displacements and modifications applied to the drawing allow the appearance of complementary concepts. Due to the deformations of the drawing, Cabri makes it possible for students to generate particular strategies. He analyses two components of the epistemology of dynamic geometry systems (DGS): in the first place, the nature of the phenomenological interface; in the second place the possible consequences on students conceptualizations, in particular the effect exerted by the 'drag mode' - the displacement of drawings on the solving of familiar geometrical problems described as "static". The author distinguishes two types of strategy: static and dynamic. A strategy is said to be static if it does not produce any movement on the different parts of a drawing; it is said to be dynamic if the solving process is based on the deformations applied. Most experiments carried on the contribution of Cabri to the learning of geometry show that they favor the development of geometrical concepts. In general, the literature in the field tends to conclude that the structure of a particular system is a key to determine the characteristics of the ìgeometryî evolved using this system. For instance (Laborde, 1992) acknowledges that the characteristics of a particular DGS can affect the process of problem solving. Yerushalmy and Chazan (1992) conclude that the learning process experienced by the students seems to transcend the goals initially set before the software had been developed, since students elaborate new definitions, new suppositions, and significant problems. The objects in Cabri can get different functions from intrinsic ones expected by designers. So Cabri favours the appearance of idiosyncratic strategies for the solving of problems. These opinions show that the interface is neither neutral nor transparent. Taking into account that Cabri is not a perfect metaphor for geometrical objects, and considering the epistemological complexity of its functioning, we believe the analysis proposed 
by Schoenfield does not cover the building of meaning of geometrical concepts that occur with the use of this system. Meiraís idea (1995, 1998), and even more of Rabardel (1995) ones, about the role the instruments are more appropriate. This leads us to analyze the activity from another point of view, studying the actions more deeply, trying to trace the emergence of geometrical knowledge during the use of the interface itself.

Pratt and Ainley (1997) equally demonstrated how the features of Cabri influence comprehension of geometric constructions. Direct manipulation of certain elements of the interface allows students to conceive a construction as a set of drawings, closer to the theoretical definition of a geometrical figure.

Duval (1998) asserts that the dynamic geometry systems are superior to paper \& pencil; he justifies this by saying that this kind of system dissociates the intention of sketching from the production of the drawing. Therefore the subjects are supposed to explicit a geometric property before sketching an object on the screen. He writes: "computers provide enormously increased possibilities of visualisation, particularly through the introduction of the aspect of movement (Ö). And because this tool dissociates the moment of intention (the subject has only to choose an instruction in primitives of a geometrical software) and the moment of production which is executed by the computer, it opens like an ëexperimentali approach in geometry." (Duval, p. 47).

This article is organized in this manner: Section 2 deals with the process sense making to mathematical concepts in situated and mediated contexts. Section 3 presents a theoretical framework built upon constructivist foundations for the analysis of educational interfaces focused on action and mediated learning of specific concepts. Section 4 discusses an example in which this framework is employed. In this section, we discuss studentís adaptation to the interface and mediated learning. Section 5 gives a conclusion about a conceptualization point of view of different geometries perspective know in the literature.

\section{Building the meaning of mathematical concepts}

The relation between the use of pedagogical materials and Mathematics learning has been studied for many years (Bishop, 1983; Clements and Battista, 1992; Duval, 1998; Kaput, 1992, Noss and Hoyle, 1996, Schwartz, 1999). In the early seventies, Bishop (1983) was already exploring the relation between the use of instruments and the evaluation subsequent to a test of special skills; he showed that a childís previous experience with pedagogical materials could lead to different levels of performance in relation to spatial abilities. In a paper about the learning of Geometry, Clements and Battista (1992) pointed out that the use of concrete 
materials could enhance the process of learning Mathematics even among older students. In the same context, Schoenfeld (1986) states that the interaction between practical (or empirical), and deductive activities leads to mutual reinforcement. He considers that the dichotomy between empiricism and deduction in Geometry is false and observes that the majority of students consider the empirical and deductive approaches as separate domains, with their own specifications to establish the validity of a result. At the end of the first term of secondary school, many of them are "inexperienced empiricists", for which the resolution of the ruler and pair of compasses problems are just a matter of trial and error. The relation between activities with pedagogic material and the construction of geometric knowledge rests upon the acknowledgement by the student of the existence of a domain of reference and of some relation between this reference and the mathematical object conveyed by the use of the instrument. This model, called epistemic fidelity, assumes the existence of a reference domain $\mathrm{W}$ that can be thought of as a representation of a mathematical idea. The objects within $\mathrm{W}$ are thought of as particular mathematical entities, and the operations applied to these objects are procedures and results that correspond to these entities. Besides, there is a symbolic system (w), which is at the same time a symbolic representation of some mathematical abstractions selected from W. The symbols in w represent entities in $\mathrm{W}$.

This analogy between reality and thought implies the supposition that individuals have a fair comprehension of the meaning of the objects and procedures in the domain of reference $\mathrm{W}$, and of their interrelations. To understand the nature of the symbolic abstraction which makes $\mathrm{w}$ functional, individuals should have a clear comprehension of the symbols and procedures in the symbolic system itself, and understand the different structures of different symbolic systems that work as representations of the same idea so as to perceive the structural morphisms between these representations and therefore be able to conceive the underlying mathematical idea. Meira criticizes this model (Meira, 1995, 1998). His critiques are directed mainly against the exterior representation functions: analyzing the production of representations (first degree functions) he found out that the production process merged with the action of problem solving itself.

He observed that the representations put down on paper had the function of supporting the subject's modeling activity at the same time that these representations were modeled by the activity. Therefore, on the one hand, the relation between the production and the use of representations, and the activity on the other hand, has a dialectical nature. Meira puts side by side the function of the internal and external representations. It is not possible to speak of a biunivocal relationship, as both representations are parts of one same plan.

\section{A constructivist framework for the analysis of educational interfaces}


In choosing and conceiving educational software, as will be shown later on, it is essential to be oriented to take into account aspects of the learning process in the selection or design of the instruments itself. Today, few HCI theories deal with the learning of concepts from the learnerís point of view (Shneiderman, 1998).

The use of a constructivist framework, based on Piaget, for the analysis and design of educational interfaces, assumes that userís actions are organized by mental schemes that represent operational knowledge and organize the activity of the subject (Piaget, 1996; Vergnaud, 1997). There are two advantages in adopting this starting point: the existence of a large amount of work on learning process (Flavell, 1992), and the existence of explicit definitions of such concepts as those of instrument and concept. For Vergnaud, the constructivist principle can be specified by the thesis that human development occurs by way of progressive adaptation of schemes to new situations. A scheme is made of four different kinds of components:

* Goals, sub goals, expectations and anticipations.

* Rules to generate action, control, information search and selection.

* Operational invariants such as concepts-in-action (objects, categories and predicates) and theorems-in-action (sentences held to be true).

* Possibilities of inference.

To establish a coherent relation between users' adaptation to the interfaces, and the consecutive learning, we propose a model able to articulate the material and the cognitive dimensions of the activity, as is the case in the use of interfaces (Gomes, 1999). To achieve this, it is necessary to analyse the users' actions with the interface as instrumental actions that implies the adoption of the concept of instrument, as defined by Mounoud (1970) and Rabardel (1995). According to Rabardel, an instrument is made of two components: the artefact, with its functionalities, and the set of schemes developed by the user.

Artefacts are artificial objects that have been invented for certain purposes. To use a specific artefact, the learner needs to develop schemes, progressively. In the analysis of educational software, the elements of the interface are themselves treated as artefacts and considered as instruments. The use of particular instruments favours the learning of specific concepts. The value of educational software increases in the same proportion as the number of operational invariants of concepts that emerges becomes greater. 
From this conception of instrument, we can tackle the problem of learning in the use of artifacts.

Table 1 shows the structure of the instrument as defined above.

\section{Table 1. Instruments Units and Sub units}

\begin{tabular}{c|c}
\hline Units & Sub units (invariants) \\
\hline Artefacts & \\
\hline \multirow{2}{*}{ Scheme } & Theorems in action \\
\cline { 2 - 2 } & Rules of action \\
\hline
\end{tabular}

In this model, and following Rabardel, the use of educational interfaces is analysed in two steps: first the adaptation of the users to the interface, a process called 'instrumental genesis', through the development of schemes in relation with different aspects of the structure of the artefact. Second, the cognitive development that takes place during the use of the instrument.

In this paper, our analysis consists in analysing conceptualization emerging in instrumental actions. The conceptualizing process consists specifically in the development of operational invariants, without which it would be impossible for the subject to pick up the relevant information, to neglect the non-relevant one and to develop rules to generate his activity in situ. As we will see in the examples given later, theorems-in-action are crucial to interpret and characterize the actions performed, even when subject does not explicitly mention them. Actually most of them are implicit, and sometimes even unconscious.

\subsection{Form of analysis}

The instrumental genesis is analysed by following the transformations of instruments units (artefacts and schemes) and sub-units (operational invariants and rules). The analysis of cognitive development takes place with the artefacts is carried out from userís point of view. Therefore it is possible to analyse the evolution of artefacts functions attributed by users when time passes. This evolution is called instrumentalization, and reflects the assimilation of the interface by the subject. The same follow up can be done in relation to the progressive adaptation of the schemes, and it allows the observation of users adaptation to the interface.

The learning process analysis is made through the inference of the operational invariants used by each student in each of the attempts. Conceptualization comes as the activity unfolds. Therefore, it appears to us that the use of Cabri in the learning of Geometry can be described more clearly if the activity of construction using instruments is punctually and carefully 
observed. Conceptualization plays the role of structuring and restructuring the instrumental schemes of the user. It is then possible to present a detailed representation of conceptualization that appears in relation to the educational interface elements (Gomes, 1999; Gomes et. al. 2003).

As we analyzed the attempts to solve the problems, we identified a set of invariants and a set of rules that were used by the students. We took into account the fact that each particular situation evolves over time. In the sequence of situations, we tried to identify the elements that constitute instruments and that play a part in making the geometrical concepts meaningful. In our perspective, the comprehension of a mathematical concept is not the a posteriori result of becoming aware of the existence of a bi-univocal correspondence between reality and the symbolic system used.

\section{Case studies}

Here we analyse the solving of problems of Geometry with two different systems of instruments (IS): the first called ëruler and compassesí, and the second "Cabri Géomètre". We observed the activities of five pairs of students of 5th grade of French school (12-13 years olds). Each pair solved a set of six problems of geometry. To illustrate the use of the framework, we present one problem resolution by two students: SA and SB. The problem posed was the following: Build the triangle $A B C$ knowing that: $A B C$ is isosceles, base [BC]; $O$ is the center of the circumscribed circle with $A B C$; and, $B$ is symmetrical to point $A$ in relation to line (d).

Before Cabri was used, we analysed how the students solved the same kind of problem, using a ruler and a pair of compasses. With these latter instruments, the students worked alone. Those that were able to solve the problem used the concept of axial symmetry.

It happens that student SA made a mistake. He began placing point B symmetrical to point A in relation to line $d$ with a graded ruler and a square ruler. To find point $\mathrm{B}$, he took the distance from A to $d$ with the compasses and placed point $\mathrm{C}$ on the intersection between the circle and line $d$. In the end, the triangle drawn by SA is isosceles, but with $\mathrm{AB}$ as its base. Student SA did not find point $\mathrm{C}$ because he had not followed the instructions. The origin of his error is due to the fact that he had started the construction taking $d$ as the axis of symmetry. Student SB drew a circle centred in $\mathrm{O}$ using the compasses, then using a square ruler he drew the mediatrix of $\mathrm{AB}$. Figure 1 shows the work of SA (left) and SB (right). 


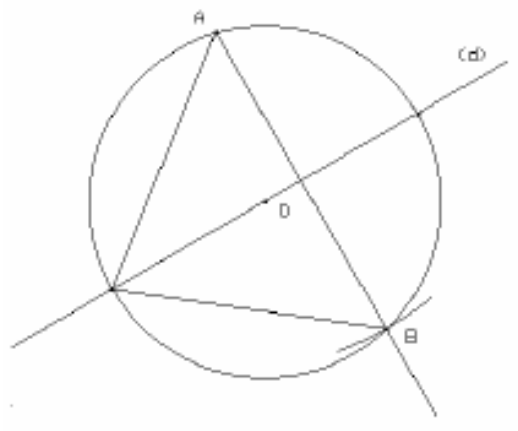

SA

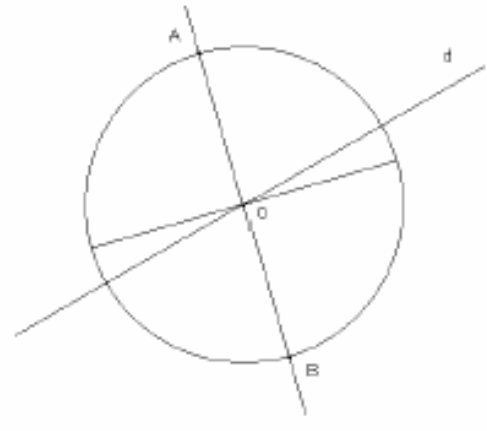

SB

Figure 1. SA and SB's drawings

Solving the problem upon presented, SA and SB performed five attempts using the Cabri software. At each step, the function of some artefacts evolved, due to the process of "instrumental genesis".

In the same way, the set of operational invariants also change from one attempt to another. In the first attempt, the students started using the central symmetry of point A, which prevented them from finding the solution. Figure 1 shows the final stage of their first drawings. During the solving process they made an attempt to solve the problem through axial symmetry; they did not succeed because they had chosen point $\mathrm{O}$ as the intersection of the perpendicular line with line d. In the ruler and compasses phase, we had seen that the students had the knowledge (operational invariants) necessary to solve it. :

* same distance of both points to any point on the axis of symmetry

* $\quad$ use of a perpendicular line to find the symmetrical point

However, they did not use these two elements but tried to find the symmetry of $\mathrm{A}$ in relation to $\mathrm{O}$, and drew a line passing through point $\mathrm{O}$ and point $\mathrm{A}$ instead of a perpendicular to $d$. After this they drew a line perpendicular to the axis, but crossing point $\mathrm{O}$ and not point $\mathrm{A}$ (dotted line on Figure 2). 


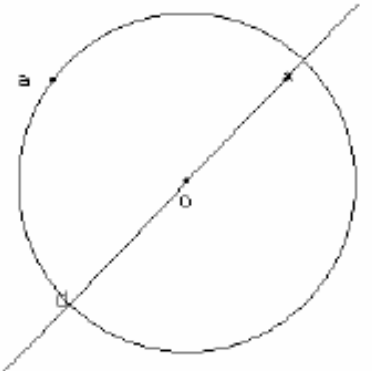

(a)

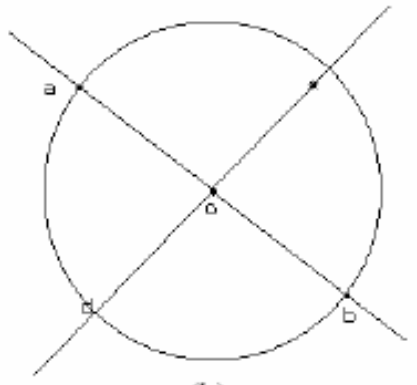

(b)

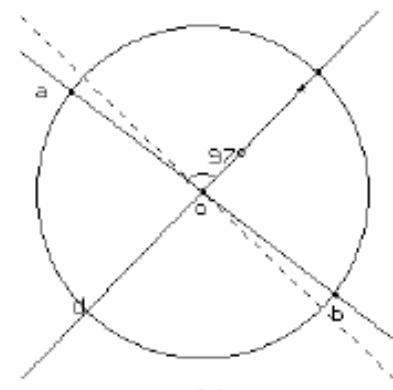

(c)

Figura 2. First attempts used by $\mathrm{SA}$ and $\mathrm{SB}$ to build an isosceles triangle

In the second attempt, the students made progress compared to the first one. They were able to find correctly point $\mathrm{B}$ symmetrical to $\mathrm{A}$ in relation to $d$, and then $\mathrm{AB}$. It is this procedure we observed closer. The students made an intermediate construction that could help them to find B. It seems that this evolution takes place owing to the scheme of instrumental action associated with the use of the pair of compasses. However, the students did not try to find point $\mathrm{C}$; they rather started again the construction. In this example, it is noteworthy that they used two circles centred on $d$. These circles are part of the new instrument used to find point $\mathrm{B}$.

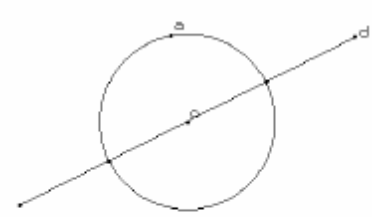

(a)

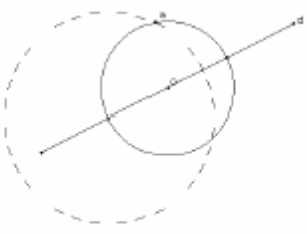

(b)

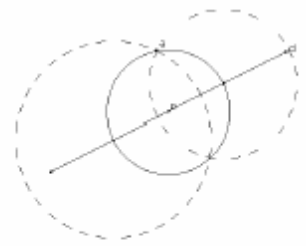

(c)

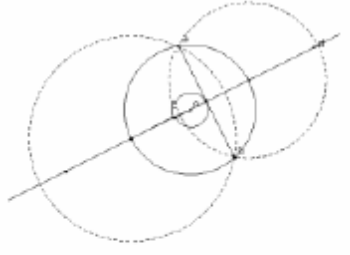

(d)

Figure 3. Second attempt

As a matter of fact this move is equivalent to the one made with a pair of compasses. The difference is that, here, the two circles have different diameters. In the construction with a pair of compasses, the students usually do not place the centres of the circles anywhere over the segment. With the pair of compasses it is expected that the identification of the point of symmetry be done with two equivalent lines.

The student's instruments did not make much progress in the third attempt. Once more, they were able to find point $\mathrm{B}$, symmetrical to $\mathrm{A}$ in relation to $d$. They tried to find point $\mathrm{C}$ but their procedure did not lead to satisfactory results. A peculiarity of this attempt is the use of an 
artifact that had not been used up to this point: the automatic measurement function of Cabri. The students employed a simpler and more effective configuration than the one used in the second attempt to find $\mathrm{B}$; in this third attempt, the circles centred on $\mathrm{d}$ are also employed. Their intersection points are therefore symmetrical in relation to $d$. The artefact is then simplified. The instrumental scheme is adapted to attain the final configuration according to the problem text. The measure of same figure parts is used to allow the student to conclude when the configuration is like attended. In so doing, the instrument was transformed and a new strategy emerges. In the same way, the conceptualization on isosceles triangle used in this attempt is quite different from that one in first attempt.

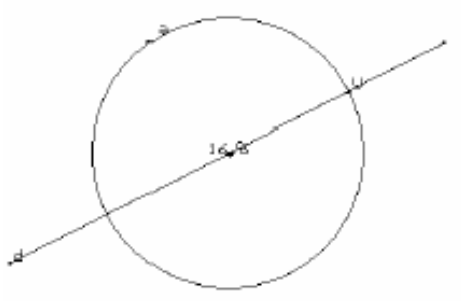

(a)

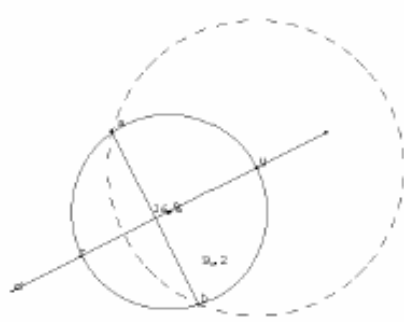

(b)

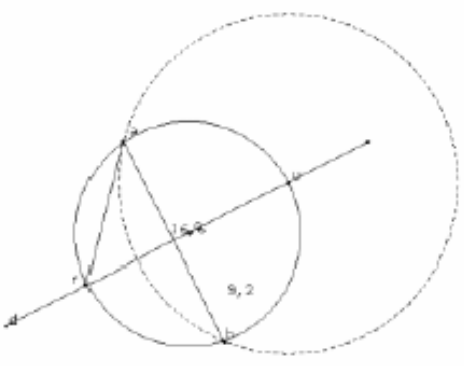

(c)

Figure 4. Third attempt

The peculiarity of the fourth attempt is that the simplification resulting from the preceding step is stabilized. We did not observe any hesitation to find the axial symmetry. The automatic measurement function is no longer used. During this attempt, the instrument is a new transformed. The way students find point B is accomplish with a single circle and a segment. The conceptualization was equally changed.

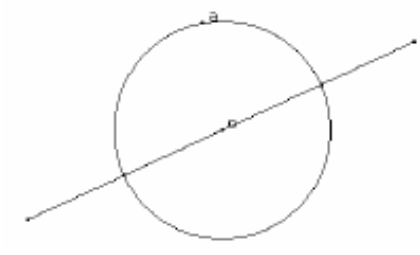

(a)

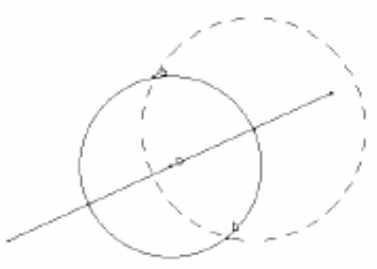

(b)

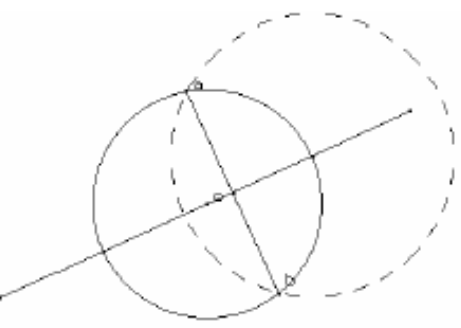

(c)

Figure 5. Fourth attempt 
Finally, SA and SB were successful to construct a figure that could resist dynamic displacement. The students built something a little different from what they had done before: they did not use a second circle to find point $\mathrm{B}$ anymore. In this attempt, they found $\mathrm{B}$ with the aid of a perpendicular line to $d$, and a single circle, described in the enunciation. The circle and the perpendicular line were used as instruments in the intermediate construction (c). The students seem to have necessary well assimilated the function of the Cabri instruction PERPENDICULAR LINE, and a new scheme is developed in association with it. This scheme coordinates the use of the drawing made with Cabri to find the symmetrical of point A and the concept of axial symmetry. They found point $C$ easily with the aid of a second circle centered in A. Therefore they had associated a function to the new circle to report a distance, a function that they had previously understood with the use of the compasses. Figure 6 shows this sequence.

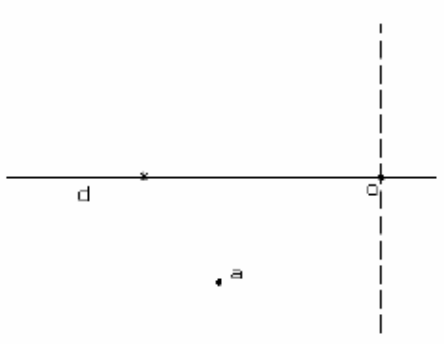

(a)

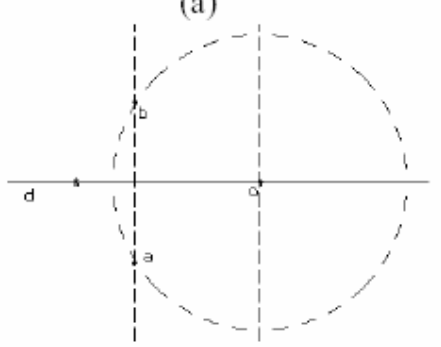

(d)

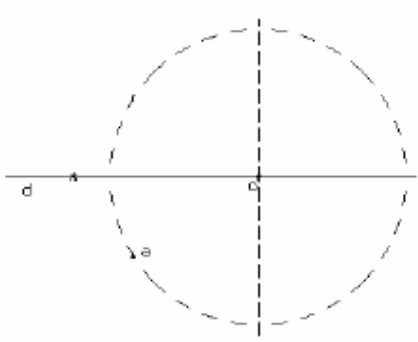

(b)

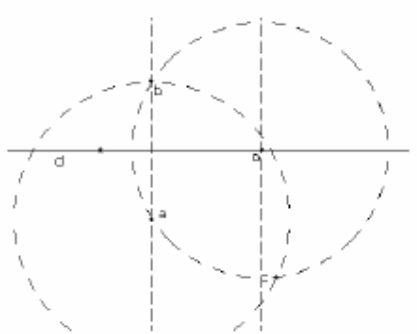

(e)

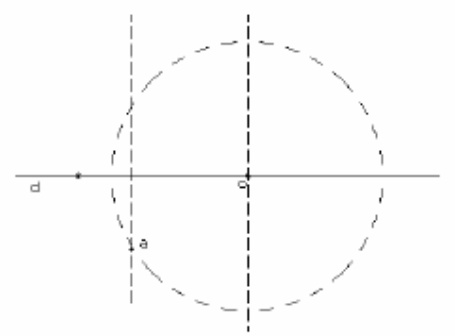

(c)

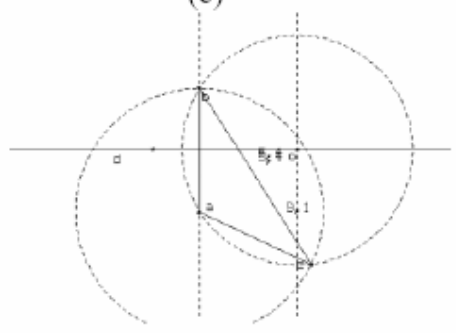

(f)

Figure 6. Fifth attempt

The line perpendicular to $d$ at point $\mathrm{O}$ seems to have no function whatsoever. The students had been guided by a procedure similar to the one used in the first attempt, when a false conceptualisation about central symmetry had been employed. They seem to be guided by the wrong idea of drawing a perpendicular line crossing $d$ at a point different from the appropriate one to find the symmetrical point of A.

\section{Results}


Until now, the analysis of only one task has been presented. We will now use the results of the analysis of all six problems. In order to present a synthetic body of knowledge underlying the activities associated with both systems of instruments, we identify a set of "elementary situations" that were used during problem-solving process. We focus our attention on some elementary situations associated with the use of the ruler and compasses and Cabri.

\subsection{Analysis of instrumental genesis}

The form of the successive instruments with Cabri primitives evolved. The students go from a configuration with three circles and a perpendicular line to $\mathrm{d}$ (second attempt) to a configuration with two new circles and a perpendicular line (third and fourth attempts) to finally reach a version with only one new circle and a perpendicular line. The use of Cabri also allowed the accommodation of the scheme of reporting a length to localize the symmetrical point of another point. The use of the ruler and a pair of compasses has probably influenced by the form students develop strategies to construct geometrical figures with Cabri, step by step. This conclusion is convergent with the one offered by other authors (Laborde \& Caponni, 1994; Pratt \& Ainley, 1997; Hölz, 1997). But we would like to go one step further.

We can observe that students acted as if they believed that a circle is centered on a second circle and as if the first radius was smaller than the second diameter. Therefore, they are secant and the two secants points with the center of the first circle define an isosceles triangle inscribed in the second circle. The composition of their artifact reflects this knowledge because we have a circle centered in $\mathrm{O}$ passing through $\mathrm{A}$ and a second circle centered in 'a' passing through $\mathrm{B}$. The intersection of both circles was placed on the Cabri drawing (Figure 6). It is clear that, in order to carry out this construction, the students needed to deal with significantly more geometrical knowledge than in any intermediate stages.

We noticed that as soon as the artifact's composition changes, there is necessarily a transformation of any rules subjacent to the artifactís organized actions. Consequently, the scheme of use associated with this instrument changes. When students pass from an empirical strategy to a correct geometrical one, the whole subjacent conceptualization changes dramatically. The schemes are not organized simply based on knowledge regarding the isosceles triangleís topology and on length variations. During a correct construction, on their third attempt, they measured the segment $[\mathrm{AB}]$, and then they drew another segment with exactly the same measurements, whenever they turned its writing end the other way, by using the SEGMENT DEF 2 PTS tool. This action is organized starting from the idea that the circle is the locus of the equidistant points of a point and that, in particular, the points of intersection between a circle and another circle centered on the first define an isosceles triangle inscribed 
within the center of the second one. The instrumental development leads possibly to a conceptual development just as learning to use CABRI results in the learning of geometrical concepts. These concepts are quite necessary in order to achieve tasks with this system.

Cabri's direct manipulation influenced the emergency of various instruments and, consequently, unlike strategies. The integration of so composite instruments produce what the literature had evidenced as the "different geometries" that emerges from the use of particular SDGs, diverse instruments systems.

\subsection{Analysis of mediate learning}

Starting from the definition of mental scheme - seen as an invariant organization of activity we proceed to the location of sequences of actions realised with a same set of artefacts or with different ones. During the process of analysis, we identify classes of elementary situations that are useful to appreciate, for example, the influence of a previous experience with artefacts during the adaptation to a new one. The determination of the type of situation is rather complex since it takes place through the indirect identification of schemes that seem to correspond to the set of situations under scrutiny. Theoretically, there are no equivalent schemes, given that adaptation is a continuous process, and a subsequent action is never identical to a previous one. This postulate is a fundamental aspect of the ìconstructivisticî dialectic theory. However, one can imagine criterions that will enable us to define some degree of similarity between two organizations of activity.

Analysing the protocols of all students we could identify the situations presented in Table

2. Situations $\mathrm{A}$ and $\mathrm{J}$ are equivalent, so are $\mathrm{D}$ and $\mathrm{K}, \mathrm{G}$ and $\mathrm{L}, \mathrm{E}$ and $\mathrm{H}$.

Table 2. Distribution of situations according to instrument systems used

\begin{tabular}{l|l}
\hline \multicolumn{1}{c|}{ Ruler and a pair of compasses } & \multicolumn{1}{c}{ Cabri Software } \\
\hline A: report in another direction a length over an axis & J: report a distance \\
D: find points equidistant to two fixed points & K: find two points equidistant to a point on the circle \\
E: find the symmetrical point of a point & H: find the symmetrical point of a point \\
G: find the middle point of a segment & L: find the middle point of a segment \\
\hline
\end{tabular}

To describe how invariants (theorems in action and rules) are used in action or emerges during problem solving activity, we organized the distribution of a certainly amount of them according to the situations. There are usually several operational invariants in the same elementary situation, and the same invariants may be relevant for several situations. Given that we can 
infers and analyse the situations, we can evaluate how an educational interfaces, a particular system of instruments, favour the emergence of specific conceptualizations.

In Table 3, we place two situations in the same column, and use symbols to indicate the system of instruments with which the invariants appear: $\mathrm{RC}$ for ruler and compasses, and $\mathrm{C}$ for Cabri. If the same invariant appear in both, we use the notation C-RC. In the first column, we indicate the concepts and theorems in action that appear during the use of at least one system of instruments: mainly axial symmetry (AS), circle (CR), triangle (TG), isosceles triangle (IT), and mediatrix (MT).

\section{Table 3. Distribution of the invariants used trough situations}

\begin{tabular}{|c|c|c|c|c|c|c|}
\hline \multicolumn{3}{|c|}{ Invariants } & \multicolumn{4}{|c|}{ Situations } \\
\hline Code & Description & Value & A- J & D-K & G-L & $\mathrm{E}-\mathrm{H}$ \\
\hline IT-01 & The adjacent sides to the superior vertex of a isosceles triangle are equal & Right & C-RC & C-RC & & \\
\hline IT-04 & The mediatrix of an isosceles triangle equals its height & Right & & $\mathrm{RC}$ & & \\
\hline TG-02 & The orthocenter of a triangle is the center of the circumscribed circle & Right & & & $\mathrm{C}$ & \\
\hline MT-01 & The points of a mediatrix of a segment are equidistant to the extremes of this segment & Right & & $\mathrm{RC}$ & & \\
\hline MT-02 & The mediatrix of an iscosceles triangle divides an iscosceles triangle in two equivalent triangles & Right & & & $\mathrm{C}$ & \\
\hline MT-04 & The mediatrix of a segment is perpendicular to this segment & Right & & & $\mathrm{C}$ & \\
\hline MT-07 & A point equidistant to the ends of a segment is located halfway between these points & Wrong & & $\mathrm{RC}$ & & \\
\hline MT-08 & $\begin{array}{l}\text { Only the intersection point between a segment and its mediatrix is equidistant to } f \text { its ends, } \\
\text { and the rest of the points of the mediatrix do not }\end{array}$ & Wrong & & $\mathrm{RC}$ & & \\
\hline AS-01 & $\begin{array}{l}\text { The symmetrical of a point } \mathrm{A} \text { in relation to a straight line belongs to a perpendicular to the } \\
\text { line crossing point } \mathrm{A}\end{array}$ & Right & & & & $\mathrm{RC}$ \\
\hline AS -02 & In axial symmetry, the distance of a point to a straight line is kept & Right & & & & $\mathrm{RC}$ \\
\hline AS -06 & $\begin{array}{l}\text { In axial symmetry, the symmetrical of a straight line is a straight line, of a segment of a } \\
\text { straight line, too, and if a straight line crosses the symmetry axis, its symmetrical crosses it at } \\
\text { the same point }\end{array}$ & Right & & & & $\mathrm{RC}$ \\
\hline AS -11 & $\begin{array}{l}\text { Given that an external point } \mathrm{A} \text {, and its symmetrical in relation to an axis, two other points on } \\
\text { the symmetry axis are equidistant to point } \mathrm{A} \text { and its symmetrical, even if the distance to these } \\
\text { points over the projection of } \mathrm{A} \text { are different }\end{array}$ & Right & & & & $\mathrm{C}$ \\
\hline AS -13 & $\begin{array}{l}\text { The symmetrical of a point in relation to the axis is colinear to the first point and to a point } \\
\text { already placed on the axis }\end{array}$ & Wrong & & & & $\mathrm{RC}$ \\
\hline AS -16 & $\begin{array}{l}\text { A point extemal to an axis, and its symmetrical are crossed by at least two circles with } \\
\text { different diameters with their centers on the symmetry axis }\end{array}$ & Right & & & & $\mathrm{C}$ \\
\hline CR-01 & A circle is the place of equidistant points to a center & Right & $\mathrm{RC}$ & & & \\
\hline CR-02 & If a circle is centered in the middle of a segment, its ends are points in the same circle & Right & & & $\mathrm{RC}$ & \\
\hline CR-05 & $\begin{array}{l}\text { If a circle has its center on a second circle, and if the radius of the first is smaller than the one } \\
\text { of the second, then they are secants, and the two points also define a isosceles triangle } \\
\text { inscribed into the second }\end{array}$ & Right & $\mathrm{C}$ & $\mathrm{C}$ & & \\
\hline
\end{tabular}

Taking a look at this table, one can immediately see that the learning provided by Cabri and by the ruler and compasses is particular. The conceptualization emerging from the use of each system of instruments is different. If it were otherwise, there would be a greater number of CRCs presented on the table. For each system, the users used different properties of the same mathematical concepts. Each interface has its own scope of value for the teaching and learning of a specific concept. Wrong operational invariants were observed only when students used rules and a pair of compasses (MT-07, MT-08 and AS-13). This system seems more flexible in use, inducting including wrong usage. On the opposite hand, Cabri's interface is more restrictive 
but not directive. This has produces a more effective relation between usage and correct invariants in action.

Operational invariants are part in schemes structuring process. From a high abstract point of view, some 'particular geometry', promoted by the use of a particular instrument system are mainly diverse. This result suggests that one could not conclude that no interface, or instrument system, is superior in relationship to other. Interface analysis should be conducted empirically. A superior interface is that promoting and demanding as more as possible different situations and invariants.

\section{Conclusions}

In this paper, we analysed the contribution of the use of Dynamic Geometry Software to the learning of Geometry from the point of view of the conceptualizing process, having Rabardel's and Vergnaud's theories as a theoretical background. We analysed the Cabri Géomètre software use, and compared its use with the use of artefacts common to the teaching of Geometry, such as rulers and pairs of compasses.

The results presented by the existing bibliography show a positive influence of such software. It is important to envisage that the use of different specific software, or systems of instruments, would favour the emergence of "different geometries". It is important to mathematics teachers and educational software designers be able to map the conceptual field promoted by the interface. To teachers, this information can support software choice, inclusion on class planning; and to designer, this is important to support their analysis on the range of the software in relation to the conceptual field explored.

Therefore, we were enabled to conclude that for each set of artefacts the students use different properties of the geometric concepts could emerge. This result corroborates the conclusion that asserts that the use of different sets of instruments would lead to the conception of ëspecific geometriesí. We come to the conclusion that "different geometries" diverge on implicit conceptualizations and specific strategies emerge from the use of specific interfaces. This conclusion implies that the teaching of geometry should be richer in situations, and should not be restricted to the use of a limited set of artefacts.

Moreover, these initial results point to the need for mapping the conceptual field promoted by each software, in order to allow educational software evaluation become a evaluation of process, and not only a measure of technical and pedagogical quality. In this way, information 
generated in this kind of evaluation can support reflections of quality and use of software programs, as well as in a catalogue of software analysis, teachers can choose a software and using them according their practice, even for software restricted to a small part of the conceptual field. The analysis showed that the software programs explore a small part of the focused field. Nonetheless, this analysis allows teachers to choose and use the software programs considering their possibilities and limitations.

From a didactical point of view, this conclusion implies that the teaching of Geometry should be rich in situations, and should not be restricted to the use of a limited set of artefacts due to the fact that the artefacts favour the emergence of a preferential subset of properties of geometric concepts.

A second contribution of this paper is the elaboration of a powerful method of analysis of educational interfaces. The method presented here can be widely used for the analysis and the evaluation of educational interfaces in general. It complements traditional approaches of the ergonomical facilities of such interfaces, adding to them the identification of different invariants and implicit conceptualization applied in action. An extrapolation of this paper results' to the design of educational interfaces is that with the methods application we can identify the correlation between interface pieces usage and learning. This can conduct the designer to implement direct manipulation effect that corresponds to the learnerís representation and the effective contribution of the interface into learning process.

\section{References}

Bishop A. J. (1983). 'Space and geometry', In R. Lesh and M. Landau (eds.) Acquisition of Mathematics concepts and processes, Academic press, p. 175-203.

Clements D. H. and Battista M. T. (1992). Geometry and spatial reasoning, In Douglas A. Grouws (Ed.) Handbook of research on Mathematics Teaching and Learning, New York, Macmillan, p. 420-64.

Duval R. (1998). Geometry from a Cognitive Point of View. In C. Mammana e V. Villani (Eds.) Perspectives on the Teaching of Geometry For the 21st Century: an ICMI study, pp.33-48, Dordrecht: Kluwer.

Duval R. (1990). Pour une approche cognitive de líargumentation, Annales de Didactique e de Sciences Cognitives, vol. 3, pp. 195-221, IREM de Strasbourg, Strasbourg. 
Flavell J. H. (1992). A Psicologia do Desenvolvimento de Jean Piaget, São Paulo: Livraria Pioneira Editora.

Gomes A. S. (1999). Développement conceptuel consécutif a l'activité instrumentée Líutilisation díun systeme informatique de géomètrie dynamique au collège, Unpublished $\mathrm{PhD}$ Thesis, UniversitÈ Paris V, Paris [www.cin.ufpe.br/ asg].

Gomes, A. S.; Queiroz, A. E. M.; Carvalho, F. A. T.; Alves, F. J. A. (2003). The Learning of geometric concepts using Dynamic Geometry Software. In: JULIE A. JACKO (ED.) \& CONSTANTINE STEPHANIDIS (ED.) HCI INTERNATIONAL 2003, Creta, Grécia, Human Computer Interaction, International Proceedings, 2003. Lawrence Erlbaum Associates.

Hölzl R., How Does 'Dragging' Affect The Learning Of Geometry, International Journal of Computers for Mathematical Learning 1: 169-187, 1996.

Kaput J. J., Technology and mathematics education, In Douglas A. Grouws (Ed.) Handbook of research on Mathematics Teaching and Learning, New York, Macmillan, p. 515-556, 1992.

Laborde C. (1992) Solving problems in computer based geometry environments: the influence of the features of the software, Zentralbaltt für Didaktik des Mathematik, 1992 (4), p. 128-35.

Laborde C. and Capponi B. (1994) Cabri Géomètre constituant díun milieu pour l'apprentissage de la notion de figure géomètrique, Recherches en Didactique des Mathématiques, vol 14, $\mathrm{n}^{\circ}$ 1.2, p. 165-210, Ed. La Pensée Sauvage, Grenoble.

Laborde C. and Vergnaud G. (1994) L'apprentissage e l'enseignement des mathématiques, In G. Vergnaud (Ed.) (1994) Apprentissages e didactiques, où en est-on?, Paris, Hachette; Meira L. (1995) The Microevolution of Mathematical Representations in Children's Activity,Cognition and instruction, 13 (2), 269-313.

Meira L. (1998) Making sense of Instructional Devices: The emergence of Transparence in Mathematical Activity, Journal for Research in Mathematics Education, vol. 29, n. 2, pp. 121142.

Mounoud P. (1970). Structuration de l'instrument chez l'enfant, Delachaux et Niestlé, Lausanne. 
Noss R. and Hoyles C. (1996) Windows on Mathematical Meanings - Learning Cultures and computers, Dordrecht: Kluwer Academic Publishers.

Nunes T. and Bryant P. (1997) Learning and teaching mathematics: An international Perspective, Psychology Press: Hove.

Piaget J. and Inhelder B. (1989) A Psicologia da Criança, Rio de Janeiro: Editora Bertrand Brasil S.A.

Piaget, J. (1996). Biologia e Conhecimento, Editora Vozes: Petropolis.

Pratt D. and Ainley J. (1997). The construction of meanings for geometric construction: two contrasting cases. International Journal of Computers for Mathematical Learning. v. 1(3) p. 293-322.

Rabardel P. (1995). Les hommes e les technologies - Approche cognitive des instruments contemporains, Paris: Armand Colins.

Schoenfeld A. H. (1992). Learning to think mathematically: Problem solving, metacognition and sense making in mathematics, In Douglas A. Grouws (Ed.) Handbook of research on Mathematics Teaching and Learning, New York, Macmillan, p. 334-370.

Schoenfeld A. H. (1986) On having and using geometric knowledge, In J. Hiebert (Ed.) Conceptual and procedural knowledge the case of mathematics, Lawrence Erlbaum Associates, Hillsdale, NJ, p. 225-63.

Schwartz J. L. (1999). Can technology help us make the mathematics curriculum intellectually stimulating and socially responsible? International Journal of Computers for Mathematical Learning 4: 99-119.

Shneiderman B. (1998). Designing the user interface: strategies for effective humancomputerinteraction, Addison.

Vergnaud G. (1997). The nature of mathematical concepts. In T. Nunes e P. Bryant (Eds.), pp. 5-28. 
Yerushalmy M. and Chazan D. (1992) Guided inquiry and geometry: Some aspects of teaching with technology, Zentralbaltt für Didaktik des Mathematik, 1992 (5), p. 172-177. 